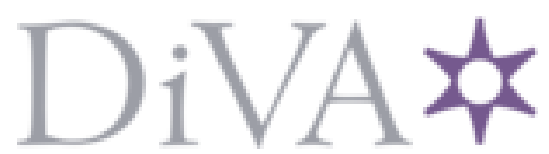

http://www.diva-portal.org

\title{
Postprint
}

This is the accepted version of a paper published in Analytical Chemistry. This paper has been peerreviewed but does not include the final publisher proof-corrections or journal pagination.

Citation for the original published paper (version of record):

Fornell, A., Nilsson, J., Jonsson, L., Periyannan Rajeswari, P., Joensson, H. et al. (2015)

Controlled lateral positioning of microparticles inside droplets using acoustophoresis.

Analytical Chemistry, 87(20): 10521-10526

http://dx.doi.org/10.1021/acs.analchem.5b02746

Access to the published version may require subscription.

N.B. When citing this work, cite the original published paper.

Permanent link to this version:

http://urn.kb.se/resolve?urn=urn:nbn:se:uu:diva-264301 
This document is confidential and is proprietary to the American Chemical Society and its authors. Do not copy or disclose without written permission. If you have received this item in error, notify the sender and delete all copies.

\section{Controlled Lateral Positioning of Microparticles inside Droplets using Acoustophoresis}

\begin{tabular}{|r|l|}
\hline Journal: & Analytical Chemistry \\
\hline Manuscript ID & ac-2015-02746v.R1 \\
\hline Manuscript Type: & Article \\
\hline Date Submitted by the Author: & 21-Sep-2015 \\
\hline Complete List of Authors: & $\begin{array}{l}\text { Fornell, Anna; Lund University, Biomedical Engineering } \\
\text { Nilsson, Johan; Lund University, Department of Biomedical Engineering } \\
\text { Jonsson, Linus; Lund University, Biomedical Engineering } \\
\text { Kumar Periyannan Rajeswari, Prem; KTH Royal Institute of Technology, } \\
\text { Science for Life Laboratory } \\
\text { Joensson, Haakan; KTH Royal Institute of Technology, Science for Life } \\
\text { Laboratory } \\
\text { Tenje, Maria; Lund University, Biomedical Engineering; Uppsala University, } \\
\text { Science for Life Laboratory }\end{array}$ \\
\hline
\end{tabular}

SCHOLARONE ${ }^{\text {Mx }}$

Manuscripts 


\title{
Controlled Lateral Positioning of Microparticles inside Droplets using Acoustophoresis
}

Anna Fornell ${ }^{1}$, Johan Nilsson ${ }^{1}$, Linus Jonsson ${ }^{1}$, Prem Kumar Periyannan Rajeswari ${ }^{2}$, Haakan N. Joensson ${ }^{2}$, and Maria Tenje ${ }^{1,3^{*}}$

${ }^{1}$ Dept. Biomedical Engineering, Lund University, Box 118, S-221 00, Lund, Sweden

${ }^{2}$ Div. of Proteomics and Nanobiotechnology, KTH Royal Institute of Technology, Science for Life Laboratory, Box 1031, S-171 21 Solna, Sweden

${ }^{3}$ Dept. Engineering Sciences, Science for Life Laboratory, Uppsala University, Box 534, S75121 Uppsala, Sweden

*E-mail: maria.tenje@angstrom.uu.se

Keywords: acoustophoresis, droplets, enrichment, lab-on-a-chip devices, microfluidics

\begin{abstract}
In this paper, we utilise bulk acoustic waves to control the position of microparticles inside droplets in two-phase microfluidic systems and demonstrate a method to enrich the microparticles. In droplet microfluidics different unit operations are combined and integrated on-chip to miniaturise complex biochemical assays. We present a droplet unit operation capable of controlling the position of microparticles during a trident shaped droplet split. An acoustic standing wave field is generated in the microchannel, and the acoustic forces direct the encapsulated microparticles to the centre of the droplets. The method is generic and requires no labelling of the microparticles, and is operated in a non-contact fashion. It was possible to achieve $2+$ fold enrichment of polystyrene beads $(5 \mu \mathrm{m}$ in diameter $)$ in the centre daughter droplet with an average recovery of $89 \%$ of the beads. Red blood cells were also successfully manipulated inside droplets. These results show the possibility to use acoustophoresis in two-phase systems to enrich microparticles, and opens up for new dropletbased assays that are not possible to perform today.
\end{abstract}




\section{INTRODUCTION}

Droplet microfluidics has been the focus of intensive research within recent years. ${ }^{1,2}$ The principle of this technology is to use two immiscible phases to create a two-phase system where each droplet, or plug, can be viewed as an independent reservoir for biological or chemical reactions. The applications and motivation for such system is to provide miniaturisation and increased throughput of different biological and chemical assays as well as a tool for material syntheses. ${ }^{3-5}$ In this work, we present a method to reduce the droplet volume while maintaining the majority of the microparticles in a pre-determined daughter droplet, by first positioning the microparticles to the centre of the droplet using bulk acoustic waves and then split the droplet into three daughter droplets.

To miniaturise complex assays on chip, several droplet "unit operations" have been developed that can be combined into highly advanced microsystems designed for specific applications. Examples of such unit operations include encapsulation of single cells inside droplets, injection of reagents into droplets, droplet splitting, and droplet sorting. ${ }^{6-9}$ However, there is still a need for a flexible unit operation to laterally position microparticles or cells in a label-free manner inside droplets. This is useful for the development of droplet washing procedures, or for microparticle enrichment inside droplets, where the final destination of the microparticles after a splitting step must be possible to control. Today, droplets are typically split using T-crosses without any positioning of the encapsulated microparticles, thus the microparticles are randomly distributed between the outlets, leading to no controlled enrichment. ${ }^{8,10}$ Some researchers have addressed this and similar topics by controlling the position of microparticles inside droplets using magnetic forces, but this technique cannot easily be transferred into label-free cell-based assays since it requires magnetic properties of the microparticles. ${ }^{11,12}$ A recent report also shows how hydrodynamics can be used to position microparticles inside droplets. ${ }^{13}$ However, that technique relies upon particle sedimentation and requires slow flows to successfully handle biologically relevant microparticles, thus 
limiting its use for high-throughput screening applications. In addition, it cannot be externally regulated on-demand.

Acoustic forces has proven a suitable miniaturised method to manipulate small particles or objects in microfluidic channels (i.e. acoustophoresis) in a non-contact fashion. ${ }^{14,15}$ The forces exerted to achieve the particle positioning are gentle, resulting in no negative side effects when handling e.g. cells. ${ }^{16}$ As opposed to other manipulation methods such as dielectrophoresis and magnetophoresis, it is unaffected by particle charge, $\mathrm{pH}$ and ionic strength of the solutions. ${ }^{14}$

In earlier work, acoustics have been applied to droplets in order to move microparticles inside sessile droplets surrounded by air, ${ }^{17,18}$ manipulate entire droplets, ${ }^{19,20}$ control droplet size, ${ }^{21}$ steer and split plugs, ${ }^{22}$ controlled merging, ${ }^{23}$ and to align cells before single cell printing with a drop dispenser; ${ }^{24}$ but this is the first time acoustic forces are used to position and enrich microparticles and cells inside moving droplets.

A comprehensive overview of the theory of acoustic manipulation of particles in microfluidic channels can be found in literature, and only the main concepts are described here. ${ }^{25}$ Acoustophoresis utilises ultrasonic standing waves to generate forces that can be used to position particles inside microsystems. By matching the frequency of the sound to the width of the microchannel, a standing wave field will be created across the channel with pressure minima and maxima at specific locations.

For a $1 \mathrm{D}$ planar standing $\lambda / 2$-wave the primary acoustic radiation force, $F^{\mathrm{rad}}$, is described by Equation (1), $F_{z}^{r a d}=4 \pi \phi(\widetilde{\kappa}, \tilde{\rho}) k a^{3} E_{a c} \sin (2 k z) \quad$ Equation 1 where $\phi$ is the acoustic contrast factor, $k$ is the wave number $(k=2 \pi / \lambda), a$ is the particle radius, $E_{a c}$ is the acoustic energy density, and $z$ is the distance from the wall. Depending on the sign of the acoustic contrast factor, particles will be moved either towards the pressure nodes or antinodes. The acoustic contrast factor relates the compressibility $(\kappa)$ and the density $(\rho)$ of 
the suspended particles $\left(\kappa_{p}, \rho_{p}\right)$ to the surrounding fluid $\left(\kappa_{0}, \rho_{0}\right)$, and is described by Equation (2).

$\phi(\widetilde{\kappa}, \tilde{\rho})=\frac{1}{3}\left(\frac{5 \widetilde{\rho}-2}{2 \widetilde{\rho}+1}-\tilde{\kappa}\right) \quad$ Equation $2 \mathrm{a}$

$\tilde{\kappa}=\frac{\kappa_{p}}{\kappa_{0}} \quad$ Equation $2 \mathrm{~b}$

$\tilde{\rho}=\frac{\rho_{p}}{\rho_{0}} \quad$ Equation 2c

Our system is operated by creating resonance between the channel walls since the two phases (in our case water and olive oil) have similar acoustic impedance, meaning that the channel is the resonance chamber. Since the channel height is $<\lambda / 2$, no vertical particle manipulation is expected. In earlier work with acoustic manipulation of particles inside sessile water droplets surrounded by air, ${ }^{17}$ the droplet itself is the resonance chamber due to the large difference in acoustic impedance between water and air. To be able to manipulate microparticles inside droplets in a two-phase system with similar acoustic impedance, essentially two requirements must be fulfilled. First, as in single-phase systems, resonance must be generated in the microchannel. The frequency to create a standing $\lambda / 2$-wave depends on the channel dimensions and the sound velocity in the fluid. In a two-phase system the dispersed phase is surrounded by the continuous phase thus, the frequency of the standing wave is dependent on the combination of sound velocities in both phases. Even if the droplets apparently fills the width of the channel there is still a thin lubricating film of the continuous phase present between the droplet and the channel wall. ${ }^{26}$ Second, high degree of coupling of the sound into the droplets must be achieved. If there is a mismatch in acoustic impedance between the two phases this will reflect and refract the sound at the dispersed/continuous phase interface. ${ }^{27}$ This indicates the importance of acoustic impedance matching between the two phases to achieve strong acoustic forces inside the droplets. 
Here, as illustrated in Figure 1, we show how acoustophoresis can be combined with droplet microfluidics to create a droplet microfluidics unit operation capable of controlling the position of microparticles during a droplet splitting step. We have operated our system at $\lambda / 2-$ resonance that positions the microparticles to the centre of the droplets, and by combining it with a trident shaped droplet split, the microparticles are directed to the centre daughter droplet during the split, here named controlled splitting. We quantify the success of this operation by reporting on microparticle recovery and microparticle enrichment factor in the centre daughter droplet. We show that acoustic focusing of microparticles in droplets can be applied both to polystyrene beads and red blood cells.

\section{EXPERIMENTAL SECTION}

\section{Microchip design}

All required steps for enrichment (i.e. droplet generation, encapsulation of microparticles, positioning of microparticles and droplet splitting) are integrated on a single microfluidic chip and performed in a continuous mode. Monodisperse aqueous droplets were generated in oil using a flow focusing geometry, where the dispersed phase entered from the centre channel and the continuous phase entered from the two side channels. The inlets and outlets had a symmetric design, and the centre and side channels were oriented $45^{\circ}$ in relation to each other. To minimise flow fluctuations the two side channels had a common fluid inlet and outlet, respectively.

\section{Microchip fabrication}

The microfluidic channels were fabricated by photolithography and anisotropic wet etching with $\mathrm{KOH}\left(40 \mathrm{~g} / 100 \mathrm{ml} \mathrm{H}_{2} \mathrm{O}, 80^{\circ} \mathrm{C}\right)$ of a $<100>$ silicon wafer. The main channel structures were oriented $45^{\circ}$ in relation to the primary flat of the wafer resulting in main channels with a rectangular cross-section with a depth of $165 \mu \mathrm{m}$ and width of $400 \mu \mathrm{m}$. Holes for fluid inlets 
and outlets were wet etched through the wafer and the chip was sealed by anodic bonding of a $1.1 \mathrm{~mm}$ thick glass lid. A piezoelectric element (Pz26, Ferroperm Piezoceramics A/S) was glued using cyanoacrylate glue (Loctite 420 , Henkel AG \& Co.) on the silicon side of the chip to ensure good acoustic coupling into the chip. The channels were treated with a surface coating agent (Repel-Silane ES, Pharmacia Biotech) to render the channels hydrophobic.

\section{Experimental set-up}

An illustration of the experimental set-up is shown in Figure 2. The piezoelectric element was actuated with a function generator (33220A, Agilent Technologies Inc.) connected via an amplifier (75A250, Amplifier Research). The frequency was matched to create $\lambda / 2$-resonance in the channel (1.80 MHz at $\left.25 \mathrm{~V}_{\text {peak-peak }}\right)$.

The fluid flows were controlled by syringe pumps (NEMESYS, Cetoni GmbH) connected to the chip via Teflon tubings. The flow in the side inlets and all the outlets were directly controlled by the syringe pumps, and the dispersed phase was introduced to the system via a submerged Teflon tube in an Eppendorf tube to ensure no pressure difference build-up in the system. The flow rates were optimised to achieve stable droplet generation and splitting. In the experiments with polystyrene beads the volumetric flow rate of the continuous phase was set at $6 \mu \mathrm{l} / \mathrm{min}$, and the flow rate of the dispersed phase was set at $12 \mu \mathrm{l} / \mathrm{min}$ by setting the flow rate in the centre outlet at $6 \mu \mathrm{l} / \mathrm{min}$ and the total flow rate in the side outlets at $12 \mu \mathrm{l} / \mathrm{min}$. In the cell experiment the volumetric flow rate of the continuous phase was set at $5 \mu \mathrm{l} / \mathrm{min}$, and the flow rates in the centre and side outlets were set at 4 and $6 \mu 1 / \mathrm{min}$, respectively. Images of the droplets were acquired with a camera (XM10, Olympus) mounted on a microscope (BX51W1, Olympus) with 4x or 10x objective.

Olive oil $\left(v_{\text {olive oil }}=1450 \mathrm{~m} / \mathrm{s}, \rho_{\text {olive oil }}=0.91 \mathrm{~kg} / \mathrm{dm}^{3}\right)$ was chosen as the continuous phase since olive oil and water $\left(v_{\text {water }}=1481 \mathrm{~m} / \mathrm{s}, \rho_{\text {vatten }}=1.00 \mathrm{~kg} / \mathrm{dm}^{3}\right)$ have similar acoustic properties. The specific acoustic impedance $(z=\rho v)$ of olive oil and water are 1.32 MRayl and 1.48 MRayl, 
respectively. Our experimental results show that the use of fluorinated oil (HFE Novec 7500, $z_{\text {HFE Novec } 7500}=1.07$ MRayl) as the continuous phase does not result in a sufficiently strong acoustic resonance in our system.

\section{Experimental procedure}

Water droplets were generated in olive oil and polystyrene beads ( $5 \mu \mathrm{m}$, FLUKA) were encapsulated ( $6 \times 10^{6}$ beads $/ \mathrm{ml}$, resulting in $\sim 475$ beads in each droplet). This concentration was chosen for easy visualization of the experiments and since it is a typical concentration found in bead-based assays. Downstream the channel, the droplets were split into three daughter droplets at a trifurcation, resulting in a splitting ratio of approximately $1 / 3$ in each channel with slightly less in the sides. This splitting step was performed with both ultrasound on and off and there was no difference noted in the volumes of the daughter droplets.

To perform quantitative analysis of recovery and enrichment, the droplets were collected in reservoir Teflon tubings with a volume of $300 \mu$ l. The droplets were transferred to new individual Eppendorf tubes and allowed to coalesce. In the Eppendorf tubes the oil and water phases form a heterogeneous solution and the oil could be removed by pipetting. The concentration of polystyrene beads in the aqueous phase collected at the centre and side outlets was counted using a microparticle counter (Multisizer 3, Beckman Coulter, Inc.).

To demonstrate the possibility to manipulate cells inside droplets, red blood cells were encapsulated inside the droplets. Blood samples were obtained from healthy volunteers under informed consent. Before droplet generation the red blood cells were transferred from whole blood to phosphate-buffered saline (PBS) via centrifugation 5 times with repeated removals and additions of $1 \mathrm{ml}$ PBS, and then diluted 200 times in PBS to obtain a suitable concentration of cells for visualization. Followed by droplets generation and acoustic manipulation of the encapsulated cells.

\section{Analysis}


The volume of the droplets was determined by measuring the cross-sectional area of the droplets using Image J software making the assumption that the droplets have the height of the channel. This yields a small and consistent overestimation of the volume due to the lubricating film between the channel and the droplets and the fact that the droplets are rounded at the front and back end.

Recovery is determined by measuring the concentrations and volume of the daughter droplets as previously described, and calculated according to Equation (3),

$$
\begin{aligned}
& \text { Recovery }=\frac{n_{\text {centre droplet }}}{n_{\text {centre droplet }}+2 n_{\text {side droplet }}} \rightarrow \\
& \frac{C_{\text {centre droplets }} V_{\text {centre droplet }}}{C_{\text {centre droplets }} V_{\text {centre droplet }}+2\left(C_{\text {side droplets }} V_{\text {side droplet }}\right)}= \\
& \frac{C_{\text {centre droplets }}}{C_{\text {centre droplets }}+\frac{2 V_{\text {side droplet }}}{V_{\text {centre droplet }}} C_{\text {side droplets }}} \quad \text { Equation } 3
\end{aligned}
$$

where $n$ is the number of microparticles in the each centre droplet $\left(n_{\text {centre droplet }}\right)$ and side droplet $\left(n_{\text {side droplet }}\right), C$ is the concentration of microparticles in the centre droplets $\left(C_{\text {centre }}\right.$ droplets $)$ and the side droplets $\left(C_{\text {side droplets }}\right), V$ is the volume of a daughter droplet in the centre outlet $\left(V_{\text {centre droplet }}\right)$ and side outlet $\left(V_{\text {side droplet }}\right)$, respectively.

The enrichment factor after droplet splitting is defined as Equation (4).

$$
\text { Enrichment factor }=\frac{C_{\text {centre droplets }}}{C_{\text {initial }}} \quad \text { Equation } 4
$$

To avoid measurement errors caused by sedimentation of the microparticles in the Eppendorf tube the initial concentration was calculated from the measured microparticle concentrations in the centre and the side droplets combined.

\section{RESULTS AND DISCUSSION}

Aqueous droplets containing polystyrene beads were generated, ultrasound was applied to the system and the frequency was optimized to create resonance. Polystyrene beads in water have 
positive acoustic contrast factor and are expected to be moved to the centre of the droplets, i.e. the pressure nodes, by the acoustic forces, which was also observed when ultrasound was activated (see the photograph in Figure 1). At the trifurcation point downstream the channel every droplet were split into three daughter droplets. The volumes of the daughter droplets in the centre and side channels were $28 \mathrm{nl}$ and $25 \mathrm{nl}$, respectively. When the acoustic forces were applied during the droplet splitting step, it was possible to direct the majority of the microparticles in the original droplet into the centre daughter droplet, while significantly fewer microparticles were collected in the side daughter droplets, see Figure 3 and supplementary film.

When droplets are transported through microfluidic channels a complex pattern of flow fields arise due to the presence of the immiscible interface between the continuous and dispersed phase. ${ }^{26,28}$ These flow fields were also observed in the system, and imply that there is a competition between the acoustic forces acting to position the microparticles to the centre of the droplets and the drag forces on the microparticles from the circulating flows inside the moving droplets. M. Hein et al. recently showed how microparticles accumulate at the sides of the droplets depending on the relation between capillary number of the system and particle sedimentation rate. ${ }^{13}$ As we operate with microparticles of lower sedimentation rate $(0.68$ $\mu \mathrm{m} / \mathrm{s})$ and higher capillary number (0.009), this effect does not play a dominant role in our system.

The concentration of microparticles in the centre and side daughter droplets was measured with and without ultrasound, and the results are presented in Table 1. The experiments were performed three times $(n=3)$, with data acquisition in triplicates. Errors are reported as one standard deviation of the measurements. A recovery of microparticles of $89 \pm 4 \%$ was achieved when ultrasound was applied, compared with only $43 \pm 2 \%$ in the negative control. This means that close to $90 \%$ of the microparticles in the original droplet were found in the centre daughter droplet after the splitting. Achieving a high recovery is 
especially important when handling scarce samples and if several consecutive controlled splitting steps would be performed.

When ultrasound was applied $2.45 \pm 0.10$ fold enrichment was achieved in the centre droplets. Even in the negative control with no ultrasound applied a small enrichment was found in the centre droplets. We measured $1.17 \pm 0.05$, indicating that the complex pattern of internal motions and aggregation of the microparticles in the droplet may play a role here. The enrichment factor is interesting to study because it also takes into account the amount of liquid removed from the original droplet, which is a critical parameter for the development of on-chip droplet washing unit operation.

The experiments were also performed with 8 times lower microparticle concentration $\left(7.5 \times 10^{5}\right.$ beads $\left./ \mathrm{ml}\right)$ resulting in similar recovery and enrichment factor, thus the initial microparticle concentration is not critical for successful system performance.

To increase enrichment further, the design of the splitting trifurcation and the flow ratio between the centre and side channels may be optimised to remove more liquid to the sides while microparticles still are retained in the centre daughter droplets. In addition, it would be beneficial to achieve stronger acoustic field by e.g. increasing the voltage to attain stronger acoustic focusing. However, when handling biological material or other heat sensitive reactions this must be balanced by the need to avoid excessive heating.

To evaluate the suitability of the described technique for droplet-based cell assays, red blood cells were used to show the possibility to manipulate cells inside droplets. Red blood cells have been used extensively in acoustofluidics systems and have positive acoustic contrast factor in PBS. ${ }^{14}$ The cells were encapsulated in PBS droplets and at activation of ultrasound the cells were positioned to the centre of droplets, as shown in Figure 4. This shows the possibility to use the presented method for biological applications. 


\section{CONCLUSIONS}

We have successfully demonstrated that acoustophoresis can be used to laterally position microparticles inside droplets, and combined it with a trident shaped droplet split to achieve enrichment of microparticles inside droplets. We have also shown that it is possible to manipulate red blood cells inside droplets. Particle enrichment and removal of medium are key parts in many standard biological assays, and we believe that the described technique will prove a useful tool that can be integrated in droplet microfluidic circuitry to be able to perform cell-based assays that are not possible to perform today.

\section{Acknowledgements}

This work was funded by the Swedish Research Council, the Crafoord Foundation, Foundation Maja and Erik Lindqvist, and Foundation Olle Engkvist Byggmästare through grants received by MT. HJ acknowledges financial support by the Swedish Research Council Formas "Strong research Environments" project No. 2011-1692, BioBridges. 


\section{REFERENCES}

(1) Seemann, R.; Brinkmann, M.; Pfohl, T.; Herminghaus, S. Rep. Prog. Phys. 2012, 75 (1), 016601.

(2) Teh, S. Y.; Lin, R.; Hung, L. H.; Lee, A. P. Lab Chip 2008, 8 (2), 198-220.

(3) Guo, M. T.; Rotem, A.; Heyman, J. A.; Weitz, D. A. Lab Chip 2012, 12 (12), 21462155.

(4) Joensson, H. N.; Andersson Svahn, H. Angew. Chem. Int. Ed. Engl. 2012, 51 (49), 12176-12192.

(5) Song, H.; Chen, D. L.; Ismagilov, R. F. Angew. Chem. Int. Ed. Engl. 2006, 45 (44), 7336-7356.

(6) Clausell-Tormos, J.; Lieber, D.; Baret, J. C.; El-Harrak, A.; Miller, O. J.; Frenz, L.; Blouwolff, J.; Humphry, K. J.; Koster, S.; Duan, H.; Holtze, C.; Weitz, D. A.; Griffiths, A. D.; Merten, C. A. Chem Biol 2008, 15 (5), 427-437.

(7) Abate, A. R.; Hung, T.; Mary, P.; Agresti, J. J.; Weitz, D. A. Proc. Natl. Acad. Sci. U. S. A. 2010, 107 (45), 19163-19166.

(8) Link, D. R.; Anna, S. L.; Weitz, D. A.; Stone, H. A. Phys. Rev. Lett. 2004, 92 (5), 054503.

(9) Schmid, L.; Weitz, D. A.; Franke, T. Lab Chip 2014, 14 (19), 3710-3718.

(10) Lagus, T. P.; Edd, J. F. J. Phys. D. Appl. Phys. 2013, 46 (11), 114005.

(11) Brouzes, E.; Kruse, T.; Kimmerling, R.; Strey, H. H. Lab Chip 2015, 15, 908-919.

(12) Lombardi, D.; Dittrich, P. S. Anal. Bioanal. Chem. 2011, 399 (1), 347-352.

(13) Hein, M.; Moskopp, M.; Seemann, R. Lab Chip 2015, 15, 2879-2886.

(14) Lenshof, A.; Magnusson, C.; Laurell, T. Lab Chip 2012, 12 (7), 1210-1223.

(15) Ding, X.; Li, P.; Lin, S.-C. S.; Stratton, Z. S.; Nama, N.; Guo, F.; Slotcavage, D.; Mao, X.; Shi, J.; Costanzo, F.; Huang, T. J. Lab Chip 2013, 13 (18), 3626-3649.

(16) Wiklund, M. Lab Chip 2012, 12 (11), 2018-2028.

(17) Oberti, S.; Neild, A.; Quach, R.; Dual, J. Ultrasonics 2009, 49 (1), 47-52.

(18) Rogers, P. R.; Friend, J. R.; Yeo, L. Y. Lab Chip 2010, 10 (21), 2979-2985.

(19) Li, S.; Ding, X.; Guo, F.; Chen, Y.; Lapsley, M. I.; Lin, S. C. S.; Wang, L.; McCoy, J. P.; Cameron, C. E.; Huang, T. J. Anal. Chem. 2013, 85 (11), 5468-5474. 
(20) Leibacher, I.; Reichert, P.; Dual, J. Lab Chip 2015, 15, 2896-2905.

(21) Schmid, L.; Franke, T. Lab Chip 2013, 13 (9), 1691-1694.

(22) Sesen, M.; Alan, T.; Neild, A. Lab Chip 2015, 15, 3030-3038.

(23) Sesen, M.; Alan, T.; Neild, A. Lab Chip 2014, 14 (17), 3325-3333.

(24) Leibacher, I.; Schoendube, J.; Dual, J.; Zengerle, R.; Koltay, P. Biomicrofluidics 2015, 9 (2), 024109.

(25) Laurell, T.; Lenshof, A.; Eds. Microscale Acoustofluidics; The Royal Society of Chemistry: Cambridge, 2015.

(26) Baroud, C. N.; Gallaire, F.; Dangla, R. Lab Chip 2010, 10, 2032-2045.

(27) Kinsler, L. E.; Frey, A. R.; Coppens, A. B.; Sanders, J. V. Fundamentals of Acoustics, 4th ed.; John Wiley \& Sons, 2000.

(28) Kurup, G. K.; Basu, A. S. Biomicrofluidics 2012, 6 (2), 022008. 


\section{FIGURES AND CAPTIONS}
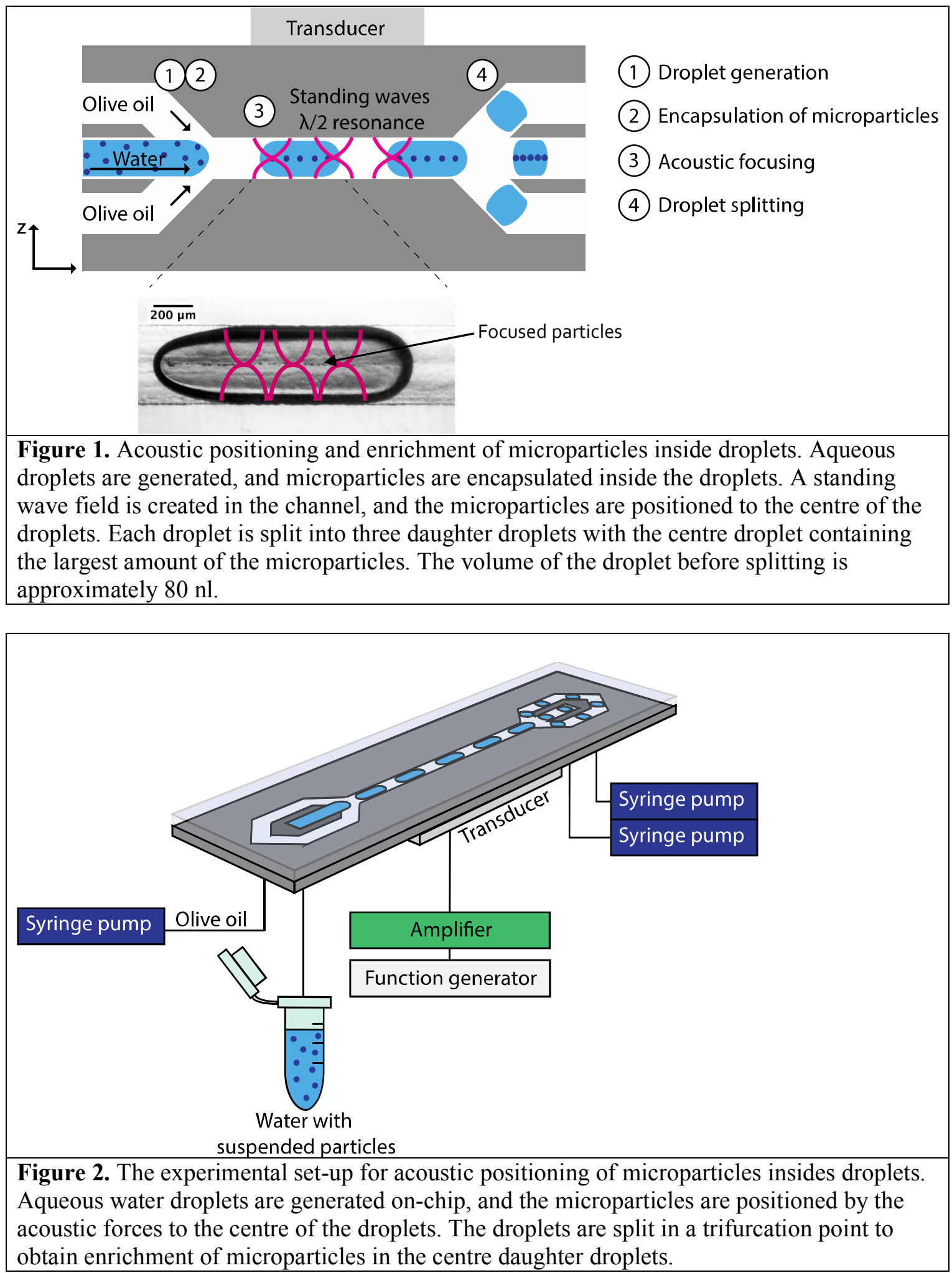


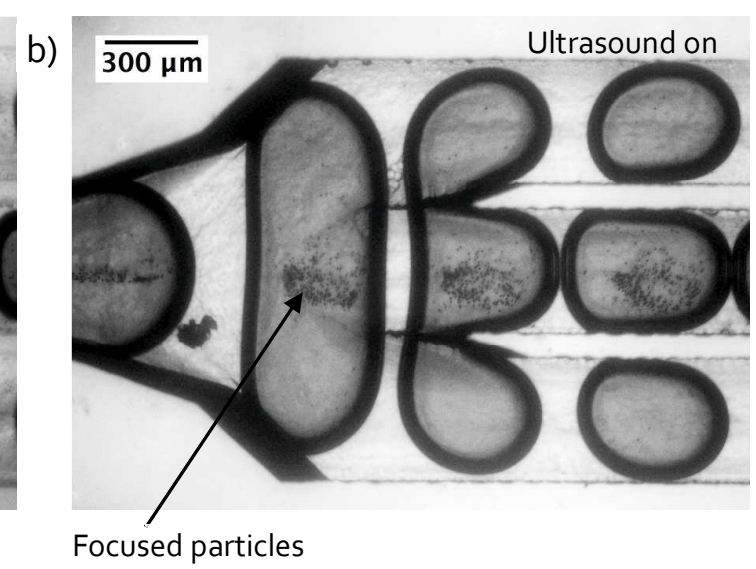

Figure 3. Acoustic positioning and enrichment of microparticles during a trident shaped droplet split. a) Without ultrasound. The microparticles are distributed in the entire main droplet, resulting in no considerable enrichment of microparticles. b) With ultrasound. The microparticles are positioned to the centre of the droplets by the acoustic forces, directing the microparticles to the centre daughter droplet after splitting, leading to a $2+$ fold enrichment of microparticles.

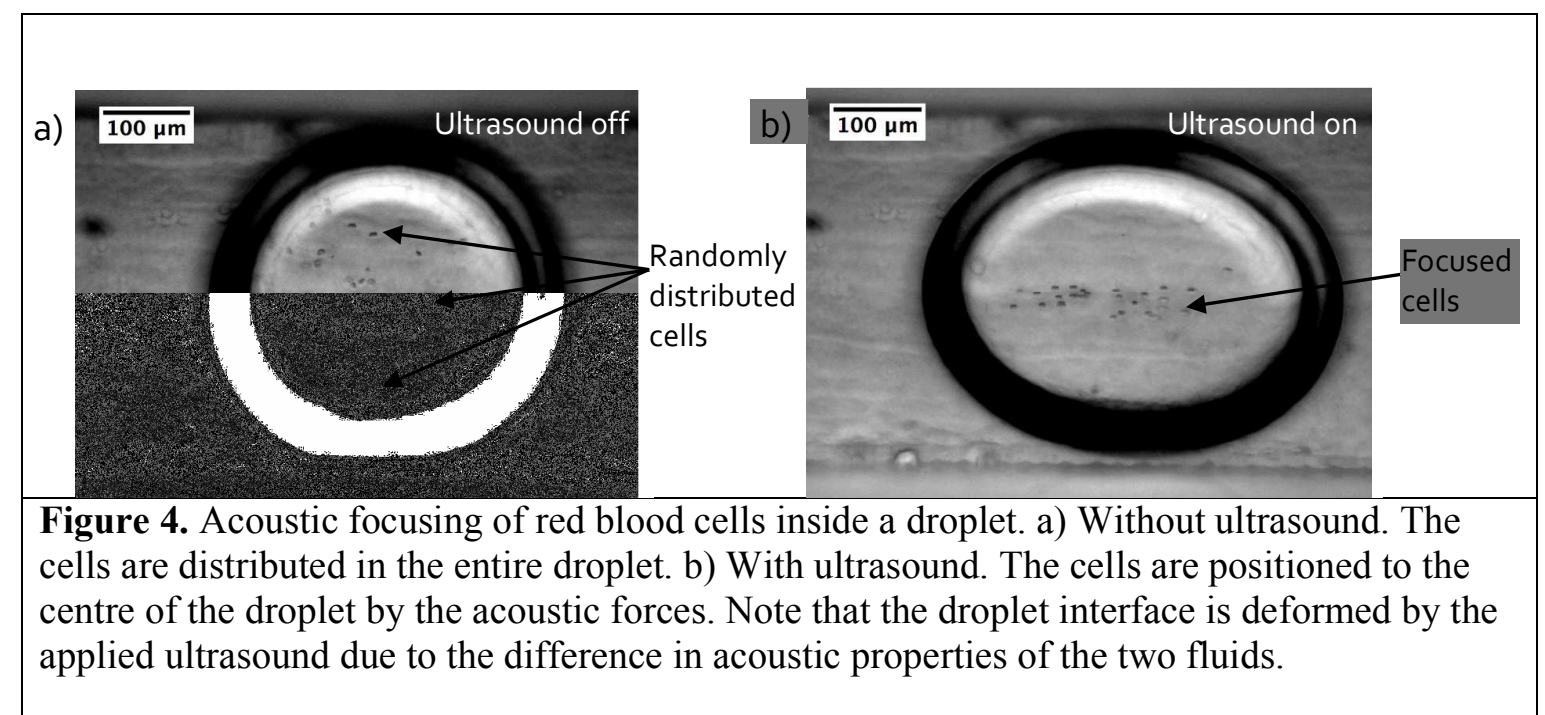


Table 1. The concentration of polystyrene beads in the centre and side daughter droplets. Errors are reported as one standard deviation of the measurements.

\begin{tabular}{||l|l|c|c|}
\hline \multicolumn{2}{|c|}{} & $C_{\text {centre droplets }}($ beads $/ \mathrm{ml})$ & $C_{\text {side droplets }}$ (beads/ml) \\
\hline \multirow{3}{*}{ Ultrasound on } & run 1 & $11.0 \times 10^{6}$ & $1.1 \times 10^{6}$ \\
\cline { 2 - 5 } & run 2 & $13.7 \times 10^{6}$ & $0.7 \times 10^{6}$ \\
\cline { 2 - 5 } & run 3 & $13.2 \times 10^{6}$ & $1.0 \times 10^{6}$ \\
\cline { 2 - 5 } & average & $12.6 \pm 1.4 \times 10^{6}$ & $0.9 \pm 0.2 \times 10^{6}$ \\
\hline \multirow{3}{*}{ Ultrasound off } & run 1 & $6.2 \times 10^{6}$ & $4.8 \times 10^{6}$ \\
\cline { 2 - 5 } & run 2 & $7.2 \times 10^{6}$ & $5.1 \times 10^{6}$ \\
\cline { 2 - 5 } & run 3 & $5.5 \times 10^{6}$ & $4.5 \times 10^{6}$ \\
\cline { 2 - 5 } & average & $6.3 \pm 0.8 \times 10^{6}$ & $4.8 \pm 0.3 \times 10^{6}$ \\
\hline
\end{tabular}



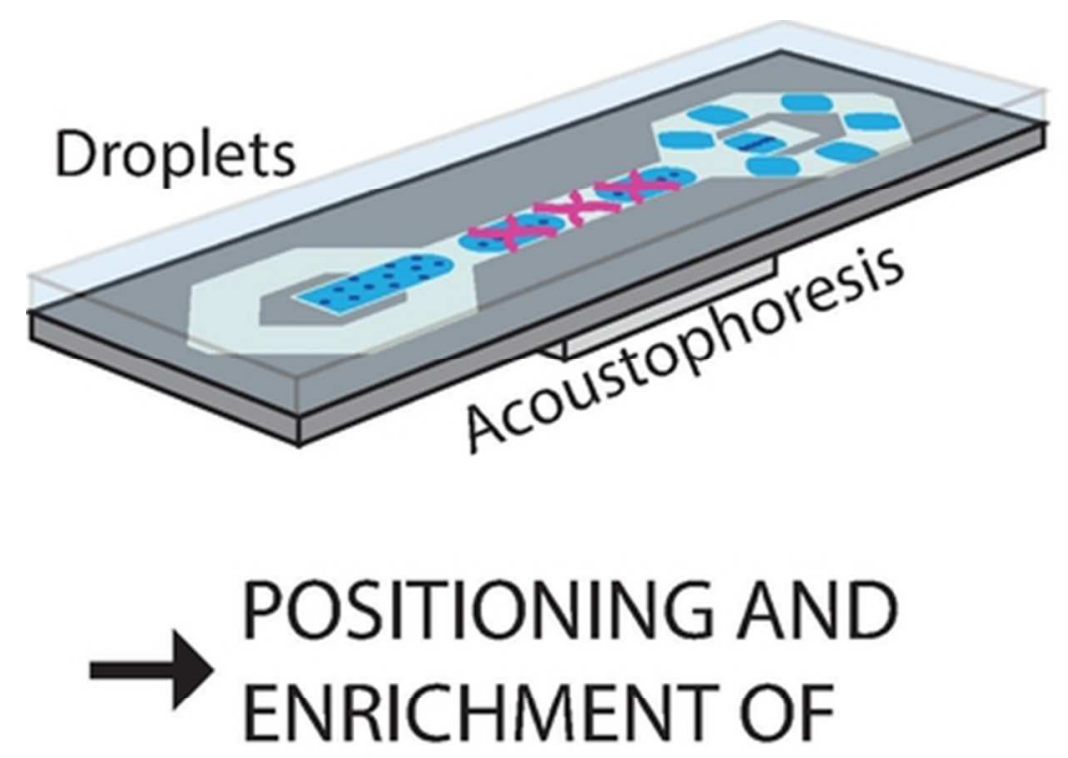
MICROPARTICLES

\author{
Table of contents figure \\ $34 \times 27 \mathrm{~mm}$ ( $300 \times 300$ DPI)
}

